\title{
METODOLOGIA DO DISCURSO DO SUJEITO COLETIVO NA AVALIAÇÃO DAS CONTRIBUIÇÕES DE UM PROJETO DE EXTENSÃ̃O UNIVERSITÁRIA
}

\author{
Lucas Soares Santos ${ }^{1}$, Claudio José dos Santos Júnior ${ }^{2}$, \\ Maria Rosa da Silva ${ }^{3}$, Sandra Adriana Zimpel ${ }^{4}$
}

\section{RESUMO}

O objetivo deste estudo foi conhecer a percepção de integrantes do Projeto de Extensão Universitária Sorriso de Plantão sobre as possíveis contribuições do referido projeto para formação profissional e de que modo a ação de extensão se vincula às estratégias de humanização da assistência à saúde. Trata-se de um estudo de abordagem qualitativa com informações obtidas a partir de entrevistas semiestruturadas e os dados analisados através da técnica do Discurso do Sujeito Coletivo. Quatro categorias foram encontradas no discurso dos acadêmicos: ser Palhaço Doutor é uma ação solidária; ser Palhaço Doutor é transformador; vivência de acolhimento, interdisciplinaridade e transformação da realidade; e humanização é cuidado e empatia. Verificou-se, nos discursos, que a participação no projeto trouxe contribuição significativa aos seus membros, não apenas no aprendizado sobre o tema atendimento humanizado ao paciente, mas também sobre seu papel na melhora da qualidade da assistência em Saúde, na percepção da importância do trabalho em equipe e acerca do uso da ludicidade no atendimento em Saúde.

Palavras-chave: Formação profissional em Saúde. Extensão comunitária. Ludoterapia. Humanização da assistência. Pesquisa qualitativa.

\section{Como citar este documento - ABNT}

SANTOS, Lucas Soares; SANTOS JÚNIOR, Claudio José dos; SILVA, Maria Rosa da; ZIMPEL, Sandra Adriana. Metodologia do discurso do sujeito coletivo na avaliação das contribuições de um projeto de extensão universitária. Revista Docência do Ensino Superior, Belo Horizonte, v. 10, e016197, p. 1-17, 2020. DOI: https://doi.org/10.35699/2237-5864.2020.16197.

\footnotetext{
${ }^{1}$ Universidade Estadual de Ciências da Saúde de Alagoas (UNCISAL), Maceió, AL, Brasil. ORCID: https://orcid.org/0000-0002-8745-793X. E-mail: soares_lucas94@hotmail.com. ${ }^{2}$ Universidade Estadual de Ciências da Saúde de Alagoas (UNCISAL), Maceió, AL, Brasil. ORCID: https://orcid.org/0000-0002-2853-1968. E-mail: claudiosantos_al@hotmail.com. ${ }^{3}$ Universidade Estadual de Ciências da Saúde de Alagoas (UNCISAL), Maceió, AL, Brasil. ORCID: https://orcid.org/0000-0001-7431-9266. E-mail: enfamariarosa@yahoo.com.br. ${ }^{4}$ Universidade Estadual de Ciências da Saúde de Alagoas (UNCISAL), Maceió, AL, Brasil. ORCID: https://orcid.org/0000-0002-6551-9888. E-mail: sandrazimpel@uol.com.br.
} 

EXTENSIÓN UNIVERSITARIA

\section{RESUMEN}

El objetivo de este estudio fue conocer la percepción de los integradores del Proyecto de Extensión Universitaria Sorriso de Plantão sobre las posibles contribuciones del referido proyecto de capacitación profesional y cómo la acción de extensión está vinculada a las políticas de humanización de la atención médica. La metodología se basó en un enfoque cualitativo, con información obtenida de entrevistas semiestructuradas y datos analizados utilizando la técnica del Discurso del Sujeto Colectivo. Se encontraron cuatro categorías en el discurso de los estudiantes: ser un Payaso Médico es una acción solidaria; ser un Payaso Médico es transformador; experiencia de recepción, interdisciplinariedad y transformación de la realidad; y la humanización es cuidado y empatía. En los discursos, se descubrió que la participación en el proyecto aportó una contribución significativa a sus miembros, no solo para aprender sobre el tema de la atención humanizada al paciente, sino también sobre su papel en la mejora de la calidad de la atención médica, en la percepción de la importancia del trabajo en equipo y sobre el uso del juego en la atención médica.

Palabras clave: Formación profesional en Salud. Extensión comunitaria. Ludoterapia. Humanización de la asistencia. Investigación cualitativa.

\section{COLLECTIVE SUBJECT'S DISCOURSE METHODOLOGY IN THE ASSESSMENT OF CONTRIBUTIONS OF AN EXTENSION UNIVERSITY PROJECT}

\section{ABSTRACT}

The aim of this study was to understand the perception of the Extension Project Sorriso de Plantão by its members; what are the main contributions of the referred project to each one (professionally), and how the extension action is linked to the humanization strategies of health care. This is a study with a qualitative approach, the information was obtained from semi-structured interviews and the data was analyzed using the Collective Subject Discourse technique. Four categories were found in the academic discourse: being a Clown Doctor is a solidary action; being a Clown Doctor is transformative; experience of welcoming, interdisciplinarity and reality transformation; and humanization is care and empathy. In both speeches, it was found that participation in the project brought a significant contribution to its members, not only in learning about the theme of humanized patient care, but also about its role in improving the quality of health care, in the perception of the importance of teamwork and the use of playfulness in health care.

Keywords: Professional training in Health. Community extension. Ludotherapy. Humanization of assistance. Qualitative research. 


\section{INTRODUÇÃO}

O Sistema Único de Saúde (SUS) foi criado para garantir, constitucionalmente, uma saúde de qualidade a toda população brasileira, carregando em si princípios e diretrizes de uma política de humanização na assistência à saúde (SANTOS JúNIOR et al., 2019). Sobre esse tema, Rego et al. (2008) afirmam que a humanização na Saúde pode ser entendida como um processo que busca a mudança da cultura institucional, fundamentada no respeito e na valorização da pessoa humana, aliando a competência técnica e tecnológica à competência ética e relacional.

O Ministério da Saúde (MS), motivado pela necessidade de melhorar a qualidade das práticas de assistência à saúde, criou, em 2000, o Programa Nacional de Humanização da Assistência Hospitalar (PNHAH). Esse programa estimulava a realização de diagnósticos situacionais e a promoção de ações humanizadas de acordo com as realidades locais (RODRIGUES; CALEGARI, 2016). Em 2003, o PNHAH foi substituído pela Política Nacional de Humanização (PNH), mais conhecida como HumanizaSUS, que mudou o patamar de alcance desse olhar, abordando também os hospitais de toda a rede SUS, definindo uma política cujo foco passou a ser, principalmente, os processos de gestão e de trabalho (MARTINS; LUZIO, 2017). Desde que foi criada, essa política acumula experiências, parceiros e produções, e tem promovido diferenças nos sujeitos, nas práticas e nos serviços de saúde.

Em vista desse cenário de incorporação de tecnologias leves aos cuidados em saúde, a formação dos profissionais desse segmento tem exigido cada vez mais uma educação qualificada, norteada no desenvolvimento de competências, visando a ampliação da clínica por meio da integralidade, da indissociabilidade entre atenção e gestão, e superação da fragmentação do cuidado ao paciente (FERREIRA; ARTMANN, 2018). A cada dia a sociedade exige mais dos profissionais da Saúde, buscando aqueles com conhecimento, habilidades práticas e tomada de decisões frente às situações reais (FREITAS et al., 2016). É nesse contexto que se destaca a importância da temática da humanização da assistência, assunto indispensável a ser abordado nas atividades assistenciais de ensino, de extensão e de pesquisa, que envolvam a formação do profissional para atuação na área de Saúde.

Baseado na quebra do estigma de que o hospital é um ambiente sombrio e triste, surgiu, no ano de 2002, o Projeto de Extensão Sorriso de Plantão, que visa trabalhar a sorrisoterapia em ambientes hospitalares do Estado de Alagoas desde a graduação. O Sorriso de Plantão é um Projeto de Extensão que fundamenta suas atividades no trabalho solidário e na busca por amenizar a dor de crianças que se encontram enfermas em instalações de hospitais de Maceió/AL e de seus acompanhantes (SILVA et al., 2016). 
Lucas Soares Santos, Claudio José dos Santos Júnior, Maria Rosa da Silva, Sandra Adriana Zimpel

A princípio, o projeto era restrito aos alunos dos cursos da área de Saúde da Universidade Federal de Alagoas (UFAL); aos poucos foi permitida a participação para os acadêmicos de outros cursos e, posteriormente, de outras universidades. Atualmente, os integrantes do projeto fazem parte de cursos de graduação da área de Saúde e áreas afins (Medicina, Enfermagem, Fisioterapia, Terapia Ocupacional, Fonoaudiologia, Nutrição, Odontologia, Serviço Social, Pedagogia), sendo recrutados mediante processo seletivo anual.

As visitas do Projeto Sorriso de Plantão são realizadas em seis hospitais de Maceió, uma vez por semana, com duração de três horas. Os integrantes do projeto caracterizam-se e dão vida ao seu Palhaço Doutor, apresentando estilo, habilidades e vocabulário relacionado à personalidade do personagem, facilitando a comunicação com o paciente. Inicialmente, é feita a leitura do prontuário, a partir da qual são discutidos aspectos relacionados às doenças e limitações que as crianças possuem, para que sejam respeitados os limites na hora de brincar. Pacientes em isolamento e UTI também são atendidos pelos palhaços, desde que haja liberação pela equipe hospitalar. O projeto conta com um portal na web (www.sorrisodeplantao.com.br) onde são apresentados à sociedade registros fotográficos e em vídeo dos 18 anos de atuação do Sorriso de Plantão em Alagoas.

Ademais, esse projeto visa promover uma formação humanizada que utiliza a figura do Palhaço Doutor, caracterizado com jaleco, adereços coloridos e nariz de palhaço, com a função de gerar momentos de alegria e entretenimento ao público por meio de suas atitudes engraçadas e de seu modo diferente de se vestir, levando a criança para o universo da fantasia (SILVA et al., 2016).

Vale destacar a parceria com a Universidade Estadual de Ciências da Saúde de Alagoas (UNCISAL), que vem reforçar os objetivos da promoção do bem-estar de crianças hospitalizadas, visando diminuir a ociosidade, aliviar a dor e o sofrimento por meio do acolhimento e atividades lúdicas interativas, que permitem vivenciar experiências peculiares à infância que foram interrompidas pelo tratamento hospitalar.

Partiu-se, neste trabalho, da premissa de que conhecer a contribuição de um projeto de extensão universitária para a formação profissional de um acadêmico, sob sua própria óptica, é imprescindível para que este possa: (i) facilitar a construção de novos paradigmas na sua formação enquanto futuro profissional de Saúde; (ii) e viabilizar reflexões sobre a formação em Saúde pautada na humanização da assistência, no trabalho interdisciplinar e em uma percepção ampliada do usuário do serviço de saúde.

Nesse aspecto, o objetivo deste estudo foi conhecer a percepção de integrantes do Projeto de Extensão Universitária Sorriso de Plantão sobre as possíveis contribuições desse 
Metodologia do discurso do sujeito coletivo na avaliação das contribuições de um projeto de extensão universitária

Lucas Soares Santos, Claudio José dos Santos Júnior, Maria Rosa da Silva, Sandra Adriana Zimpel

programa para formação profissional e de que modo a ação de extensão se vincula às estratégias de humanização da assistência à saúde.

\section{MÉTODOS}

Trata-se de um estudo qualitativo, de caráter descritivo e exploratório. Foram incluídos todos os acadêmicos da área de Saúde que atuaram como Palhaços Doutores no Projeto de Extensão Sorriso de Plantão nos anos de 2017 e 2018. Foram excluídos participantes que permaneceram menos de 12 meses nas atividades do projeto, que não desejaram fazer parte da pesquisa e/ou que optaram por desistir do estudo. O número de entrevistas foi definido durante a coleta de dados, mais especificamente, quando houve a saturação do tema pela repetição das falas dos acadêmicos entrevistados, conforme recomendado por Minayo (2017).

Os participantes foram convidados pelo pesquisador, e aqueles que aceitaram assinaram o Termo de Consentimento Livre e Esclarecido (TCLE) e o Termo de Autorização para Gravação de Voz (TAGV). Foram agendadas, previamente, entrevistas individuais, conforme disponibilidade do participante voluntário da pesquisa. A coleta contemplou perguntas abertas, de resposta livre, a fim de que os membros respondessem de acordo com suas percepções e da maneira que melhor acreditassem expressar sua visão sobre o tema proposto (MINAYO, 2010).

O roteiro de entrevista semiestruturada foi elaborado pelos próprios autores com base em revisão prévia da literatura sobre a atuação do Palhaço Doutor no ambiente hospitalar. Assim, a entrevista foi norteada pelas seguintes perguntas:

1. O que é ser Palhaço Doutor para você?

2. Qual a contribuição que a participação neste projeto pode ter na sua formação profissional?

3. Quais as lições de um Palhaço Doutor sobre cuidado humanizado em Saúde?

As entrevistas individuais foram gravadas em áudio, na íntegra, permitindo-se a verbalização livre, sem correções do entrevistado. Foi realizada a transcrição das falas e todos os pesquisadores envolvidos no estudo fizeram a leitura dos conteúdos. Os conteúdos gravados foram arquivados em sigilo e codificados por números para garantir a confidencialidade dos sujeitos. Além disso, visando garantir o anonimato dos participantes, as falas dos integrantes entrevistados foram identificadas pela sigla "Dr." ou "Dra.", seguido por nomes fictícios.

Após sua realização, as entrevistas foram estudadas, interpretadas e analisadas por meio da técnica do Discurso do Sujeito Coletivo (DSC), metodologia proposta no final da década de 1990 por Lefèvre e Lefèvre (2003). Trata-se de um procedimento metodológico próprio de pesquisas sociais empíricas com foco qualitativo, utilizando uma estratégia discursiva, 
Lucas Soares Santos, Claudio José dos Santos Júnior, Maria Rosa da Silva, Sandra Adriana Zimpel

visando tornar mais clara uma representação social presente no discurso, que é o modo como as pessoas pensam.

O DSC é um jogo entre 'como' e 'o que' as pessoas pensam coletivamente, pois se busca reconstruir, com pedaços de discursos individuais, como em um quebra-cabeça, tantos discursos-síntese quantos se julgue necessários para expressar um pensar coletivo sobre um fenômeno. O DSC utiliza quatro figuras metodológicas chamadas ExpressõesChave (ECH), Ideias Centrais (IC), Ancoragem (AC) e o Discurso do Sujeito Coletivo (DSC) (LEFÈVRE; LEFÈVRE, 2003).

Assim, as entrevistas foram analisadas seguindo as etapas propostas pelo DSC, conforme os seguintes passos:

- Primeiro: copiamos integralmente o conteúdo transcrito das entrevistas de cada sujeito na coluna ECH.

- Segundo: identificamos, em cada resposta, as ECH das IC e as ECH das AC, quando estas estiveram presentes. No processo de seleção das $\mathrm{ECH}$, foi retirado o discurso não relevante para a pesquisa.

- Terceiro: identificamos e descrevemos as IC e as AC com base em cada ECH colocando-as nas colunas correspondentes.

- Quarto: agrupamos as IC com o mesmo sentido, com sentido equivalente ou complementar e as separamos em categorias.

- Quinto: criamos, para cada categoria, uma IC síntese que expressasse, da melhor maneira possível, todas as IC e AC com o mesmo sentido, com sentido equivalente ou complementar.

- Sexto passo: construímos o DSC, utilizando os textos transcritos das entrevistas separados por categorias. 
Lucas Soares Santos, Claudio José dos Santos Júnior, Maria Rosa da Silva, Sandra Adriana Zimpel

Objetivo: Compreender a percepção de graduandos da área de Saúde sobre as possíveis contribuições do projeto Sorriso de Plantão para formação profissional e de que modo o projeto se vincula às estratégias de humanização da assistência à saúde.

População: Universitários da área de Saúde que participam dos ciclos 2017 e/ou 2018 do Projeto de Extensão Universitária Sorriso de Plantão, aqui denominados “Palhaços Doutores".

Critérios de Inclusão: Todos os membros com pelo menos 1 ano de atuação no projeto.

Critérios de Exclusão: Não disponibilidade para participação na pesquisa; desistência do estudo; menos de 12 meses de participação nas atividades do Projeto de Extensão.

Total de entrevistados: 20 Palhaços Doutores.

Técnicas: Entrevista semiestruturada; gravação e transcrição na íntegra das entrevistas; técnica do Discurso do Sujeito Coletivo.

Quadro 1 - Delineamento do Estudo.

Fonte: elaborado pelos autores, 2020.

A pesquisa foi aprovada pelo Comitê de Ética em Pesquisa (CEP) da Universidade Estadual de Ciências da Saúde de Alagoas (UNCISAL), sob o número CAEE: 79548717.5.0000.5011 e realizada no período de março de 2018 a fevereiro de 2019, junto aos Palhaços Doutores do referido projeto.

\section{RESULTADOS E DISCUSSÃO}

Realizou-se um total de 20 entrevistas com acadêmicos participantes do Projeto de Extensão Universitária Sorriso de Plantão em Maceió/AL. Permitiu-se evidenciar a saturação dos temas pela repetição das falas dos acadêmicos, desta feita, a etapa das entrevistas foi concluída.

A partir das entrevistas, identificou-se o perfil dos acadêmicos participantes do estudo, sendo: 16 do sexo feminino (80\%) e 04 do sexo masculino (20\%); $13(65 \%)$ maiores de 21 anos, 18 (90\%) estudantes de universidade pública, distribuídos desde o segundo até o último ano do curso de graduação.

Corroborando com esta pesquisa, o estudo de Rufinoni (2015) descreve que o número de mulheres que ingressam no ensino superior é elevado comparado ao número de homens. Além disso, a participação feminina no projeto pode estar relacionada ao relato de Handy et al. (2004), que afirmam que estas ações seriam típicas de um perfil feminino, que possuem um foco no cuidado, no conforto, no bem-estar emocional e na redução da ansiedade dos seus familiares.

Após a análise das entrevistas e identificação das IC e das AC, as que tiveram o mesmo sentido, sentido equivalente ou mesmo complementar foram agrupadas em categorias, assim nominadas: 

A. Ser Palhaço Doutor é uma ação solidária;
B. Ser Palhaço Doutor é transformador;
C. Vivência de acolhimento, interdisciplinaridade e transformação da realidade;
D. Humanização é cuidado e empatia.

A seguir, apresentamos cada um dos discursos formulados em cada categoria e suas respectivas discussões.

\section{A - Ser Palhaço Doutor é uma ação solidária}

O primeiro aspecto questionado aos acadêmicos foi a respeito do que é para ele "Ser Palhaço Doutor?". Na análise das suas respostas foram encontradas as categorias A, B, C e D - dentre essas, uma trata da atuação no projeto como ação solidária e de doação (QUADRO 2):

\begin{tabular}{|l|}
\hline DSC1: Ser Palhaço Doutor é uma ação solidária \\
\hline Ser Palhaço Doutor é doar algo que eu não sabia que eu \\
tinha dentro de mim... É participar de forma altruísta e \\
solidária, estabelecendo, na maioria das vezes, uma relação \\
entre corpo e mente. É conseguir levar alegria às pessoas, é \\
conseguir levar amor. Sem desejar e sem esperar receber \\
nada em troca, trabalhando o voluntarismo, a solidariedade. \\
Para mim, ser Palhaça Doutora foi poder doar algo que eu \\
não sabia que eu tinha dentro do meu interior.
\end{tabular}

Quadro 2 - DSC 1: Ser Palhaço Doutor é uma ação solidária.

Fonte: elaborado pelos autores, 2020.

O discurso descrito acima demonstra a compreensão dos integrantes quanto a um dos objetivos do Projeto Sorriso de Plantão, que é a promoção do bem-estar de crianças hospitalizadas, contribuindo de forma altruísta para o alívio da dor e do sofrimento, através do acolhimento e interação através de atividades lúdicas, que permitem que as experiências da internação não corrompam a infância da criança (SILVA, 2019).

O sentimento descrito pelos entrevistados reafirma o relato de Sato et al. (2016) sobre a participação do Palhaço Doutor no ambiente hospitalar transformando o artista no espelho do ser humano. Isso, então, proporciona um olhar humanizado, através da interação intersubjetiva com os usuários, ressaltando ainda que com a presença do Palhaço é possível transformar o ambiente hospitalar, trazendo benefícios não só para os pacientes, mas para todos que estiverem inseridos no ambiente. 
B - Ser Palhaço Doutor é transformador

A segunda categoria encontrada nas respostas dos acadêmicos retrata o sentimento de transformação que eles sentem ao incorporarem o personagem de Palhaço Doutor, demonstrando o quanto esse personagem facilita o acesso à criança e o quanto essas ações do projeto lhe fazem bem (QUADRO 3):

\begin{tabular}{|c|}
\hline DSC 2: Ser Palhaço Doutor é transformador \\
\hline $\begin{array}{l}\text { Ser Palhaço Doutor é participar efetivamente do tratamento } \\
\text { daquelas pessoas, porém de forma diferente. É deixar meu } \\
\text { eu mais sensível, mais delicado, mais humano transparecer. } \\
\text { É poder reavivar a criança que existe em mim, que é algo } \\
\text { que acontece de forma natural. Quando a gente coloca } \\
\text { aquele nariz é como se a gente tivesse superpoderes. Muitas } \\
\text { vezes, você está triste, mas coloca o nariz, a maquiagem, o } \\
\text { jaleco e você se transforma em uma outra pessoa, uma } \\
\text { pessoa completamente sem problemas da vida, uma pessoa } \\
\text { que está ali disposta a se doar. }\end{array}$ \\
\hline
\end{tabular}

Quadro 3 - DSC 2: Ser Palhaço Doutor é transformador. Fonte: elaborado pelos autores, 2020.

A participação no Projeto de Extensão Sorriso de Plantão trouxe aos seus integrantes uma experiência diferente da prática profissional hospitalar, corroborando com o estudo de Teixeira (2012), que relata que é importante diversificar os campos de aprendizagem para que o conhecimento não esteja voltado apenas para o fator fisiopatológico, mas para a realidade sociocultural da população na qual o estudante, e futuro profissional, será inserido.

Os Palhaços Doutores, além de proporcionarem momentos de alegria para as crianças, sentem-se felizes com sua participação no cuidado, desenvolvendo sua criatividade e sua espontaneidade (CAVALCANTE et al., 2016). Nessa mesma perspectiva, Silva e Silveira (2011) enfatizam que o profissional de Saúde que utiliza recursos lúdicos no ambiente hospitalar facilita a criação do vínculo com a criança. Para os autores, mesmo que esses recursos sejam simples atitudes que possam divertir, há uma maior interação das crianças com o profissional, bem como humanização do atendimento, mesmo que esse vínculo não esteja bem fortalecido.

No que tange à integralidade e à humanização do cuidado nas práticas de saúde, é necessário priorizar as tecnologias leves, fundamentadas no acolhimento, no diálogo, na criação de vínculo, na corresponsabilidade e na escuta entre o profissional e o paciente, 
Metodologia do discurso do sujeito coletivo na avaliação das contribuições de um projeto de extensão universitária

Lucas Soares Santos, Claudio José dos Santos Júnior, Maria Rosa da Silva, Sandra Adriana Zimpel

sendo possível perceber na fala dos alunos a criação de laços afetivos entre eles e as crianças, contribuindo na comunicação e resolução dos problemas enfrentados por eles (JORGE et al., 2011).

\section{C - Vivência de acolhimento, interdisciplinaridade e transformação da realidade}

Esta terceira categoria abordou a percepção dos integrantes sobre as possíveis contribuições que a participação no projeto de extensão trouxe para sua formação profissional. Seu discurso deixa evidenciado que os Palhaços Doutores conseguem perceber, na prática, a influência e o impacto do projeto na formação profissional, onde puderam vivenciar a atuação do trabalho em equipe, a importância do acolhimento ao paciente e aos seus familiares e a capacidade de transformar a realidade onde ele irá atuar (QUADRO 4):

DSC 3: Vivência de acolhimento, interdisciplinaridade e transformação
da realidade
Nesse projeto aprendi a importância de trabalhar em equipe, o que é
interdisciplinaridade, a aprender com o saber do outro, aprender
sobre saúde através da própria pessoa e através do paciente. Aprendi
sobre acolhimento e como transformar um ambiente em saúde. Como
profissional, eu sei que eu vou ter outro olhar pelas crianças, mas,
principalmente, vou ter outro olhar com os cuidadores, os pais, as
avós, seja lá quem for que tiver cuidado da criança. A criança que
sofre sozinha, todo mundo sofre.

Quadro 4 - DSC 3: Vivência de acolhimento, interdisciplinaridade e transformação da realidade.

Fonte: elaborado pelos autores, 2020.

Os discursos descritos evidenciam que os integrantes do Sorriso de Plantão acreditam que a participação no projeto contribuiu para sua formação profissional, corroborando com Lima et al. (2014) num estudo em que os estudantes reconheceram que o ensino exclusivamente técnico não responde às expectativas da formação profissional em Saúde. Tais dados estão igualmente em concordância com outros autores que reforçam a necessidade da adoção de políticas e ações que propiciem aos profissionais atender às expectativas dos pacientes e seus familiares para a prestação de uma assistência humanizada (CALEGARI; MASSAROLLO; SANTOS, 2015; RODRIGUES; CALEGARI, 2016; VASCONCELOS et al., 2016; MARTINS; LUZIO, 2017).

Estudos sobre interdisciplinaridade apontam que a proposta de integração entre as profissões beneficia principalmente o paciente, pois ele passará a ser compreendido como um ser completo, já que nenhuma categoria, por si só, dará conta de todas as suas necessidades. Seguindo essa lógica, o estudo de Silva e Silveira (2011) relata que o 
Lucas Soares Santos, Claudio José dos Santos Júnior, Maria Rosa da Silva, Sandra Adriana Zimpel

profissional de Saúde deve ser consciente quanto aos aspectos relacionados ao cuidado humanizado, sabendo reconhecer o ser humano na sua integridade e de forma singular. Percebe-se que esta compreensão é claramente descrita pelos participantes do projeto em questão (SALES et al., 2011; MOTA et al., 2012).

Em seus estudos, luamoto et al. (2012) e Silva, Santos Júnior e Ribeiro (2019) ressaltaram a importância de acadêmicos pertencentes às áreas de Saúde terem, desde o momento da sua entrada na graduação, uma formação voltada para a valorização do trabalho multidisciplinar, indo além do tratamento da doença e visualizando a trajetória do paciente, buscando a melhoria da qualidade da assistência. Reforçando essa afirmação, Takahagui et al. (2014) trazem como resultado de seu estudo, realizado exclusivamente com estudantes de Medicina que atuaram como Doutores Palhaços, a melhora nas suas habilidades de comunicação e na visão integral do paciente com a experiência do projeto.

Para Correia, Taveira e Freitas (2017) deve haver o investimento curricular das universidades, voltado às áreas de ciências sociais, humanas, artes e literatura, uma vez que tais áreas, sendo associadas ao estudo disciplinar, possibilitem o desenvolvimento da relação com os sujeitos. Tal conhecimento contribuirá com a percepção dos limites e as potencialidades pertinentes a cada campo, havendo, assim, uma abertura para o fazer coletivo dos profissionais da Saúde.

Os relatos encontrados na presente pesquisa reforçam os achados do estudo de Arroyo e Rocha (2010), que afirmam que as relações da sociedade com a universidade através da extensão universitária contribuem com a formação do indivíduo em duplo sentido: voltandose para os profissionais que atuarão em diversos setores da sociedade, para os membros da comunidade, para o público de modo geral e para quem estão sendo destinadas as ações extensionistas, visando contribuir com a cidadania.

Segundo Rodrigues et al. (2013), para inovar os conhecimentos, é necessário aprimorar instantaneamente o antigo e aprender o novo, o que pode ser experimentado durante extensão universitária, quando os acadêmicos saem da sua rotina em sala de aula e passam a praticar o que está proposto, aproximando as pessoas e oferecendo uma assistência de qualidade.

Porto (2017), em seu estudo, ressaltou que as participações dos acadêmicos em ações extensionistas contribuem para a qualidade do processo de formação e atuação profissional, permitindo a reflexão sobre os aspectos que precisam ser observados e, assim, visando o aprimoramento da política de extensão da instituição de ensino. 
D - Humanização é cuidado e empatia

A quarta categoria identificada nas entrevistas foi a resposta sobre quais conhecimentos sobre cuidado humanizado eles adquiriram durante sua participação no Projeto Sorriso de Plantão (QUADRO 5):

DSC 4: Humanização é cuidado e empatia

O Palhaço Doutor consegue ampliar o conceito de cuidado. Ele trabalha o cuidar, que vai além do momento do tratamento, passando desde a abordagem do paciente, da família, até a despedida. Aprende que, em todo momento, deve haver essa sensibilização para com o paciente e o acompanhante. A gente aprende a observar mais o outro, a se colocar no lugar do outro, a ter realmente empatia. É muito fácil o professor falar que precisamos ser humanizados, precisamos ser isso, precisamos ser aquilo entre quatro paredes, lá na sala é só teoria. Essa vivência como Palhaço Doutor realmente sai dessa teoria e parte para a prática da humanização.

Quadro 5 - DSC 4: Humanização é cuidado e empatia.

Fonte: elaborado pelos autores, 2020.

Os discursos encontrados comprovam a contribuição do projeto na formação do discente acerca do tema humanização na assistência à saúde. Reforçando o entendimento de humanização vivenciado em projetos de extensão, Araújo, Silva e Puggina (2007) relatam que, para que haja o atendimento humanizado, é preciso aliviar a dor e o sofrimento do outro, seguindo como base o respeito e a sua autonomia, bem como sua dignidade, tratando com solidariedade e compreendendo, assim, a dimensão do paciente e do seu acompanhante.

O estudo de Bertoncello e Pivetta (2015) relata a importância do graduando aprender a se comunicar e interagir com os pacientes, além de dar atenção aos familiares e relacionar-se com os demais profissionais de Saúde, trabalhando em equipe e tendo também capacidade de liderança e aprendizado contínuo ao longo de sua vida.

Em consonância com a fala dos entrevistados sobre o aprendizado do tema humanização da assistência à saúde, o estudo sobre Palhaços Doutores de Takahagui et al. (2014) concluiu que a maioria dos estudantes que procuram um Projeto de Extensão busca o aprimoramento pessoal e profissional, bem como humanização na relação pessoal com o doente, buscando desenvolver habilidades de comunicação para contribuir na criação do vínculo terapeuta-paciente. 
Lucas Soares Santos, Claudio José dos Santos Júnior, Maria Rosa da Silva, Sandra Adriana Zimpel

Atualmente a realidade que se observa é que os profissionais concluem sua graduação amplamente aptos tecnicamente para realizar intervenções e procedimentos, porém, demonstram fragilidade na realização do atendimento humanizado e ampliado ao usuário do serviço de saúde. Em contraponto, sabe-se que a humanização da assistência à saúde é tema de extrema relevância para os profissionais da área (MUNIZ; BARROS; NEVES, 2017; REIS-BORGES; NASCIMENTO; BORGES, 2018).

Portanto, é necessário que o tema esteja inserido nas matrizes curriculares e para além dela, ou seja, no currículo paralelo, durante toda a formação acadêmica dos profissionais, propondo discussões sobre o processo de atendimento humanizado ao paciente, aos seus familiares e até mesmo acerca das próprias relações entre os profissionais (CARNIEL; MENDES; SÁ, 2018). É preciso salientar, ademais, que, além de ter um eixo/módulo voltado para a humanização da assistência, deve-se incluir esse tema nas abordagens teóricas e práticas no processo ensino-aprendizagem nos diversos cursos de graduação da área de Saúde.

\section{CONSIDERAÇÕES FINAIS}

Neste estudo discutimos sobre o discurso de acadêmicos que participaram do Projeto de Extensão Universitária Sorriso de Plantão. Os relatos mostraram que a participação no projeto trouxe contribuição significativa aos seus membros, não apenas no aprendizado sobre o tema atendimento humanizado ao paciente, mas também sobre seu papel na melhora da qualidade da assistência em Saúde, na percepção da importância do trabalho em equipe e humanizado e acerca do uso da ludicidade no atendimento em Saúde.

Ficou evidente a percepção do aluno quanto ao seu papel, enquanto Palhaço Doutor, no cuidado humanizado e a relevante contribuição da atividade extensionista para sua formação. Ressaltamos, pela fala dos integrantes do projeto, a necessidade de as universidades proporcionarem aos seus alunos experiências de cuidado humanizado em Saúde durante a graduação, de modo que eles possam vivenciar, na prática, uma assistência diferenciada aos pacientes, para fins de crescimento profissional e pessoal.

Acreditamos que uma excelente oportunidade de viabilizar atividades práticas exitosas, como o Sorriso de Plantão, nos currículos dos cursos da área de Saúde, é através da Curricularização da Extensão, estratégia prevista no Plano Nacional de Educação (PNE), regulamentada pela Resolução no7 MEC/CNE/CES, de 18 de dezembro de 2018, e que introduz a obrigatoriedade do cumprimento de $10 \%$ de carga horária mínima dos cursos de graduação dedicada às atividades de extensão universitária. 
Metodologia do discurso do sujeito coletivo na avaliação das contribuições de um projeto de extensão universitária

Lucas Soares Santos, Claudio José dos Santos Júnior,

Maria Rosa da Silva, Sandra Adriana Zimpel

\section{REFERÊNCIAS}

ARAÚJO, M. M. T.; SILVA, M. J. P.; PUGGINA, A. C. G. A comunicação não-verbal enquanto fator iatrogênico. Revista da Escola de Enfermagem da USP, São Paulo, v. 41, n. 3, p. 419425, set. 2007. DOI: http://dx.doi.org/10.1590/S0080-62342007000300011.

ARROYO, D. M. P.; ROCHA, M. S. P. M. L. Meta-avaliação de uma extensão universitária: estudo de caso. Avaliação: Revista da Avaliação da Educação Superior, Campinas e Sorocaba, v. 15, n. 2, p. 135-161, jul. 2010. DOI: https://doi.org/10.1590/S1414-40772010000200008.

BERTONCELLO, D.; PIVETTA, H. M. F. Diretrizes curriculares nacionais para a graduação em Fisioterapia: reflexões necessárias. Cadernos de Educação, Saúde e Fisioterapia, Campo Grande, v. 2, n. 4, p. 71-83, jan. 2015. DOI: https://doi.org/10.18310/2358-8306.v2n4p71.

BRASIL. Ministério da Educação. Conselho Nacional de Educação. Resolução no 7, de 18 de dezembro de 2018. Estabelece as Diretrizes para a Extensão na Educação Superior Brasileira e regimenta o disposto na Meta 12.7 da Lei no 13.005/201. Brasília, 2018. Disponível em: http://portal.mec.gov.br/index.php?option=com_docman\&view=download\&alias=104251rces007-18\&category_slug=dezembro-2018-pdf\&Itemid=30192. Acesso em 22 jun.2020.

CALEGARI, R. C.; MASSAROLLO, M. C. K. B.; SANTOS, M. J. Humanização da assistência à saúde na percepção de enfermeiros e médicos de um hospital privado. Revista da Escola de Enfermagem da USP, São Paulo, v. 49, n. 2, p. 42-47, 2015. DOI:

https://doi.org/10.1590/S0080-623420150000800006.

CARNIEL, F.; MENDES, G. B.; SÁ, N. R. Humanização no atendimento na percepção dos profissionais de Enfermagem. Revista Interdisciplinar, Teresina, v. 11, n. 1, p. 51-63, 2018.

CAVALCANTE, A. L. et al. Sorriso de Plantão: a percepção da criança sobre o processo de hospitalização antes e após a atuação do palhaço doutor. Caderno de Graduação, Maceió, v. 3, n. 2, p. 135-148, 2016.

CORREIA, D. S.; TAVEIRA, A. G. M. M.; FREITAS, D. A. Clínica ampliada: as oportunidades de aprendizagem discente no estágio rural em Arapiraca. In: SAMPAIO, J. F. et al. (org.). A Extensão Universitária na Formação em Saúde. Maceió: Edufal, 2017, p. 87-94.

FERREIRA, L. R.; ARTMANN, E. Discursos sobre humanização: profissionais e usuários em uma instituição complexa de saúde. Ciência \& Saúde Coletiva, Rio de Janeiro, v. 23, p. 14371450, 2018. DOI: https://doi.org/10.1590/1413-81232018235.14162016.

FREITAS, M. A. O.; CUNHA, I. C. K. O.; BATISTA, S. H. S. S.; ROSSIT, R. A. S. Teaching in health: perceptions of graduates of a Nursing specialization course. Interface: Comunicação, Saúde, Educação, Botucatu, v. 20, n. 57, p. 427-436, abr./jun. 2016. DOI:

https://doi.org/10.1590/1807-57622015.0391. 
Metodologia do discurso do sujeito coletivo na avaliação das contribuições de um projeto de extensão universitária

Lucas Soares Santos, Claudio José dos Santos Júnior,

Maria Rosa da Silva, Sandra Adriana Zimpel

HANDY, F.; MOUND, R.; VACCARO, L. M.; PROCHASKA, K. Promising practices for volunteer administration in hospitals. Toronto: Canadian Centre for Philanthropy, 2004.

IUAMOTO, L. R. et al. Extensão médica acadêmica: um projeto da Faculdade de Medicina da Universidade de São Paulo para treinamento clínico e humanização do cuidado em saúde de alunos da Medicina, Nutrição e Fisioterapia. Revista de Medicina, São Paulo, v. 91, n. 3, p. 194-197, 2012. DOI: https://doi.org/10.11606/issn.1679-9836.v91i3p194-197.

JORGE, M. S. B.; PINTO, D. M.; QUINDERÉ, P. H. D.; PINTO, A. G. A.; SOUSA, F. S. P.; CAVALCANTE, C. M. Promoção da Saúde Mental - Tecnologias do Cuidado: vínculo, acolhimento, co-responsabilização e autonomia. Ciência \& Saúde Coletiva, Rio de Janeiro, v. 16, n. 7, p. 3051-3060, 2011. DOI: https://doi.org/10.1590/S1413-81232011000800005.

LEFÈVRE, F.; LEFÈVRE, A. C. O discurso do sujeito coletivo: um novo enfoque em pesquisa qualitativa. Caxias do Sul: Educas, 2003. 256p.

LIMA, C. C. et al. Humanidades e humanização em Saúde: a literatura como elemento humanizador para graduandos da área da Saúde. Interface: Comunicação, Saúde, Educação, Botucatu, v. 18, n. 48, 2014. DOI: https://doi.org/10.1590/1807-57622013.0708.

MARTINS, C. P.; LUZIO, C. A. HumanizaSUS policy: anchoring a ship in space. Interface: Comunicação, Saúde, Educação, Botucatu, v. 21, n. 60, p. 13-22, 2017. DOI:

https://doi.org/10.1590/1807-57622015.0614.

MINAYO, M. C. S. O desafio do conhecimento: pesquisa qualitativa em Saúde. 8. ed. São Paulo: Hucitec, 2010.

MINAYO, M. C. S. Amostragem e saturação em pesquisa qualitativa: consensos e controvérsias. Revista Pesquisa Qualitativa, São Paulo, v. 5, n. 7, p. 1-12, 2017.

MOTA, G. M. et al. A percepção dos estudantes de graduação sobre a atuação do "doutor palhaço" em um hospital universitário. Revista Brasileira em Promoção da Saúde, Fortaleza, v. 25, n. 2, p. 25-32, abr./jun. 2012.

MUNIZ, H. P.; BARROS, M. E. B.; NEVES, C. E. A. B. Processos de formação em Saúde: a estratégia da educação a distância numa experiência da Política Nacional de Humanização do SUS. Cadernos de Psicologia Social do Trabalho, São Paulo, v. 20, n. 2, p. 129-142, 2017. DOI: http://dx.doi.org/10.11606/issn.1981-0490.v20i2p129-142.

PORTO, V. F. A. A extensão universitária e a formação profissional em cursos de graduação. 2017. Dissertação (Mestrado em Ensino na Saúde) - Faculdade de Medicina, Universidade Federal de Alagoas, Maceió, 2017.

REGO, S.; GOMES, A. P.; SIQUEIRA-BATISTA, R. Bioética e humanização como temas transversais na formação médica. Revista Brasileira em Educação Médica, Brasília, v. 32, n. 4, p. 482-491, 2008. DOI: https://doi.org/10.1590/S0100-55022008000400011. 
Metodologia do discurso do sujeito coletivo na avaliação das contribuições de um projeto de extensão universitária

Lucas Soares Santos, Claudio José dos Santos Júnior, Maria Rosa da Silva, Sandra Adriana Zimpel

REIS-BORGES, G. C.; NASCIMENTO, E. N.; BORGES, D. M. Impacto da Política Nacional de Humanização na Estratégia Saúde da Família e na Rede de Saúde. Distúrbios da Comunicação, São Paulo, v. 30, n. 1, p. 194-200, 2018. DOI: https://doi.org/10.23925/21762724.2018v30i1p194-200.

RODRIGUES, A. C.; CALEGARI, T. Humanização da assistência na unidade de terapia intensiva pediátrica: perspectiva da equipe de Enfermagem. Revista Mineira de Enfermagem, Belo Horizonte, v. 20, 2016. DOI: http://www.dx.doi.org/10.5935/1415-2762.20160003.

RODRIGUES, A. L. L. et al. Contribuições da extensão universitária na sociedade. Cadernos de graduação, Aracaju, v. 1, n. 16, p. 111-222, mar. 2013.

RUFINONI, P. Mudanças nos programas educacionais abriram espaço para as mulheres. Portal Brasil. 2015. Disponível em: http://portal.mec.gov.br/component/tags/tag/35388-diainternacional-da-mulher. Acesso em: 04 maio 2020.

SALES, K. N. A. et al. PET-Saúde: formando discentes multiplicadores - relato de experiência. ABENO, Londrina, v. 11, n. 2, p. 51-56, 2011.

DOI: https://doi.org/10.30979/rev.abeno.v11i2.64.

SANTOS JÚNIOR, C. J. et al. Educação médica e formação na perspectiva ampliada e multidimensional: considerações acerca de uma experiência de ensino-

aprendizagem. Revista Brasileira em Educação Médica, Brasília, v. 43, n. 1, p. 72-79, 2019. DOI: https://doi.org/10.1590/1981-52712015v43n1rb20180141.

SATO, M. et al. Palhaços: uma revisão acerca do uso dessa máscara no ambiente hospitalar. Interface: Comunicação, Saúde, Educação, Botucatu, v. 20, n. 56, p. 123-134, 2016. DOI: https://doi.org/10.1590/1807-57622015.0178.

SILVA, I. D.; SILVEIRA, M. F. A. A humanização e a formação profissional em Fisioterapia. Ciência \& Saúde Coletiva, Rio de Janeiro, v. 16, n. 1, p. 1535-1546, 2011. DOI: https://doi.org/10.1590/S1413-81232011000700089.

SILVA, J. V. S.; SANTOS JÚNIOR, C. J.; RIBEIRO, M. C. Ensino integrado em Saúde e prática interprofissional: uma experiência na graduação. Revista Docência do Ensino Superior, Belo Horizonte, v. 9, p. 1-14, 17 dez. 2019. DOI: https://doi.org/10.35699/2237-5864.2019.14820.

SILVA, M. R. et al. O sorriso como recurso terapêutico à criança hospitalizada: lições de palhaços doutores para um cuidado humanizado. Cadernos de Graduação/Ciências Biológicas e da Saúde, Maceió, v. 3, n. 2, p. 43-56, 2016.

SILVA, M. R. Conhecendo o Sorriso de Plantão. 2019. Disponível em: http://www.sorrisodeplantao.com.br/. Acesso em: 29 abr. 2020.

TAKAHAGUI, F. M.; MORAES, E. N. S.; BERALDI, G. H.; AKAMINEL, G. K.; BASILE, M. A.; SCIVOLETTO, S. MadAlegria: estudantes de Medicina atuando como doutores-palhaços: 
Metodologia do discurso do sujeito coletivo na avaliação das contribuições de um projeto de extensão universitária

Lucas Soares Santos, Claudio José dos Santos Júnior, Maria Rosa da Silva, Sandra Adriana Zimpel

estratégia útil para humanização do ensino médico? Revista Brasileira de Educação Médica, Rio de Janeiro, v. 38, n. 1, p. 120-126, jan./mar. 2014. DOI: https://doi.org/10.1590/S010055022014000100016 .

TEIXEIRA, R. C. Aderência dos cursos de Fisioterapia da região Norte às Diretrizes Curriculares Nacionais. Fisioterapia em Movimento, Curitiba, v. 25, n. 1, p. 47-54, jan./mar. 2012. DOI: https://doi.org/10.1590/S0103-51502012000100005.

VASCONCELOS, M. F. F.; NICOLOTTI, C. A.; SILVA, J. F.; PEREIRA, S. M. L. R. Entre políticas (EPS - Educação Permanente em Saúde e PNH - Política Nacional de Humanização): por um modo de formar no/para o Sistema Único de Saúde (SUS). Interface: Comunicação, Saúde, Educação, Botucatu, v. 20, n. 59, p. 981-991, 15 ago. 2016. DOI:

https://doi.org/10.1590/1807-57622015.0707.

\section{AGRADECIMENTOS}

Ao Projeto Sorriso de Plantão, à UFAL e à UNCISAL.

\section{Lucas Soares Santos}

Fisioterapeuta pela Universidade Estadual de Ciências da Saúde de Alagoas (UNCISAL).

soares_lucas94@hotmail.com

\section{Claudio José dos Santos Júnior}

Biólogo e mestrando em Ensino na Saúde na Universidade Estadual de Ciências da Saúde de Alagoas (UNCISAL).

claudiosantos_al@hotmail.com

\section{Maria Rosa da Silva}

Enfermeira, doutoranda em Saúde Pública (USP), mestre em Ensino na Saúde e professora da Universidade Estadual de Ciências da Saúde de Alagoas (UNCISAL).

enfamariarosa@yahoo.com.br

\section{Sandra Adriana Zimpel}

Fisioterapeuta, doutora em Ciências, mestre em Ensino na Saúde, especialista em Docência do Ensino Superior e professora da Universidade Estadual de Ciências da Saúde de Alagoas (UNCISAL).

sandrazimpel@uol.com.br 